\title{
Faceting via correlated disorder of a stochastically growing interface or domain boundary
}

\author{
Igor F. Lyuksyutov* \\ Department of Physics, Texas A\&M University, College Station, Texas 77843-4242
}

H.-U. Everts

Institut für Theoretische Physik, Universität Hannover, Appel Strasse 2, D-30167, Hannover, Germany

(Received 11 August 1997)

\begin{abstract}
We consider a stochastically growing or evaporating interface in the presence of disorder which is correlated in the direction normal to the interface. The growth or evaporation rate at randomly distributed disorder points is assumed to be different from that of the rest of the interface. This model is of relevance not only to island growth in overlayers, but also to the domain growth in an ultrathin magnetic film after reversal of the magnetization. For a growing one-dimensional interface or a moving domain wall in a magnetic film on a crystal surface, this type of correlated disorder simulates the effect of, e.g., surface steps or grain boundaries on the growth process while, for a growing or evaporating crystal surface, it describes the effect of screw dislocations or of grain boundaries again. We show that, for interface dimensions $d=1,2$ during the growth (or evaporation) of an initially flat interface, large-scale faceting develops, although on a small scale the interface is rough. Exploiting the formal connection between the interface model and the model used in the problem of flux line localization in a superconductor we show that correlated disorder localizes the flux line in the presence of point disorder. [S0163-1829(98)03003-3]
\end{abstract}

\section{INTRODUCTION}

Studies of crystal growth, equilibrium crystal shapes, etc., date back to the beginning of this century (see, e.g., Ref. 1). Recently, a strong increase of theoretical activity in this field has been stimulated by the work of Kardar, Parisi, and Zhang (KPZ) on kinetic roughening. ${ }^{2}$ Many growth models can be mapped onto the Kardar-Parisi-Zhang (KPZ) equation. A well-known example is the so-called single-step model ${ }^{3,4}$ which describes the stochastic growth of a one-dimensional interface. General reviews on recent developments in the field can be found, e.g., in Refs. 6 and 7. Most of these works deal with the growth of three-dimensional structures (see e.g., Refs. 8-10). However, in recent years experimental observations using scanning tunneling microscopy have also revealed a large amount of interesting information about the growth of two-dimensional structures on surfaces (see, e.g., Refs. 8 and 11). Another important area of application is a magnetic overlayer. The magnetization reversal in an ultrathin magnetic film proceeds via domain growth, and it can be shown (see, e.g., Refs. 12-14) that domain growth in a twodimensional (2D) Ising model at low temperatures is described by the single-step model.

No surface, regardless of how carefully it has been prepared, is free of defects of different origin and dimensionality (point and linear). The list includes impurities segregated onto the surface, adsorbed impurities, excessive atoms of the crystal proper, steps, etc. At low temperatures, the mobility of these defects is extremely small, and they can be treated as frozen. ${ }^{15}$ These nonequilibrium defects give rise to various random fields on the crystalline surface. The lower the dimensionality of the system, the stronger the effects of frozen defects. Theoretically, the influence of frozen surface disorder on the growth processes in overlayers remains virtually unexplored.
We study the growth of islands or of magnetic domains on a substrate with correlated frozen disorder. In growing overlayers this type of disorder can be caused by surface steps or by grain boundaries. Disorder induces an inhomogeneous distribution of the growth probability on the surface. In the case of an ultrathin magnetic film, surface steps can change the magnetic domain growth probability. ${ }^{16}$

We investigate the case of stochastic (kinetic) structure growth, when the probability of the interface growth at a given point is fixed. To study this problem, we apply the single-step model (see, e.g., Ref. 5) with additional correlated disorder. This model can be described by the KPZ equation with an additional term which describes correlated disorder (see Ref. 17). This last equation can be treated analytically by using renormalization group methods and by numerical simulation. ${ }^{17}$ The aim is to obtain the growth profile and the nonequilibrium island shape on, e.g., a stepped substrate.

We generalize the approach developed to describe twodimensional island growth to higher dimensions. We use it to describe bulk crystal growth or evaporation with correlated disorder. In this case correlated disorder can be due to, e.g., screw dislocations or grain boundaries oriented normally to the surface. Nanoparticles which "float" on the surface during growth or evaporation may also be an interesting realization of correlated disorder (see, e.g., Refs. 18 and 19).

The growth of an interface in the presence of a single defect site has been considered in Ref. 17 for both 1D and 2D interfaces. The general case of many defect sites can be mapped onto the problem of a directed polymer or a flux line in disordered media. The faceting phenomenon which is the main result of our study corresponds to flux line localization in a superconductor with so-called columnar (correlated) disorder. We discuss this relation briefly in Sec. V. 
In Sec. II we introduce the model we use. Its renormalization-group ( $R G)$ analysis will be discussed in Sec. III. There we also derive qualitative results from the scaling analysis, and we establish useful relations which characterize interface faceting. Our analytic results are supported by numerical simulations which will be presented in Sec. IV.

\section{MODEL}

The simplest model to study the influence of correlated disorder is the single-step model (see, e.g., Refs. 3-5), which describes the stochastic growth of a one-dimensional interface. In this model, the interface can grow by one unit cell at a given point with fixed probability $p$ if both neighboring cells are filled. Under this condition the growing surface cannot develop overhangs (for a review, see Ref. 5). To include frozen disorder, we modify the single-step model by allowing the growth probability $p$ to depend on the interface coordinate. In the continuum limit the kinetics of this model is described by the KPZ equation with an additional term which represents the correlated disorder (see Ref. 17). For arbitrary dimensions, this modified KPZ equation has the form

$$
\frac{\partial h}{\partial t}=\nu \Delta h+\lambda(\nabla h)^{2}+\eta(\mathbf{x}, t)+\sigma(\mathbf{x}) .
$$

Here $h(\mathbf{x}, t)$ is the height of the actual interface at the coordinate $\mathbf{x}$ of the flat surface, $\lambda$ is the interface tension, and $\eta(\mathbf{x}, t)$ is a random growth probability. The effect of disorder which is correlated in the direction normal to the surface is contained in the growth rate $\sigma(\mathbf{x})$, a time-independent random function of $\mathbf{x} . \eta(\mathbf{x}, t)$ and $\sigma(\mathbf{x})$ are assumed to be Gaussian distributed with zero mean and with the correlators

$$
\begin{gathered}
\overline{\eta(\mathbf{x}, t) \eta\left(\mathbf{x}^{\prime}, t^{\prime}\right)}=2 \mathcal{D} \delta^{d}\left(\mathbf{x}-\mathbf{x}^{\prime}\right) \delta\left(t-t^{\prime}\right), \\
\overline{\sigma(\mathbf{x}) \sigma\left(\mathbf{x}^{\prime}\right)}=2 \mathcal{C} \delta^{d}\left(\mathbf{x}-\mathbf{x}^{\prime}\right) .
\end{gathered}
$$

The parameter $\mathcal{C}$ is proportional to the linear defect density $\vartheta, \mathcal{C} \propto \vartheta$. If the growth probability is the same for all points of the growing interface, the interface position is described by the correlator (rough interface)

$$
\left\langle\left[h(\mathbf{x}, t)-h\left(\mathbf{x}^{\prime}, t^{\prime}\right)\right]^{2}\right\rangle \propto\left|\mathbf{x}-\mathbf{x}^{\prime}\right|^{2} \chi f\left[\frac{\left|t-t^{\prime}\right|}{\left|\mathbf{x}-\mathbf{x}^{\prime}\right|^{z}}\right],
$$

where the exponents $\chi(d)$ and $z(d)$ are known exactly in $d=1, \chi=\frac{1}{2}$, and $z=\frac{3}{2},{ }^{20}$ and numerically in $d=2, \chi=0.387$, and $z=1.613 .{ }^{21} \chi(d)$ and $z(d)$ are connected by the scaling relation (see, e.g., Ref. 21)

$$
\chi+z=2 \text {. }
$$

\section{SCALING ANALYSIS}

Renormalization-group treatment: To derive the renormalization-group equations for Eq. (1) we use the approach which applied by Kardar, Parisi, and $Z_{\text {hang }}^{2}$ to the KPZ equation. Using the standard procedure, ${ }^{2}$ we obtain the following flow equations:

$$
\begin{aligned}
& \frac{d}{d l} \nu=\left[z-2+A_{d} \frac{2-d}{4 d} \overline{\lambda^{2}}\right] \nu, \\
& \frac{d}{d l} \mathcal{D}=\left[z-d-2 \chi+A_{d} \frac{\overline{\lambda^{2}}}{4}\right] \mathcal{D}, \\
& \frac{d}{d l} \lambda=[z+\chi-2] \lambda, \\
& \frac{d}{d l} \mathcal{C}=\left[2 z-2 \chi-d+A_{d} \frac{\overline{\lambda^{2}}}{4}\right] \mathcal{C} .
\end{aligned}
$$

Here $A_{d}=\left[2^{d-1} \pi^{d / 2} \Gamma(d / 2)\right]^{-1}$ and $\overline{\lambda^{2}}=\lambda^{2} \mathcal{D} / 2 \nu^{3}$. The first three RG equations coincide with those for the ordinary KPZ equation. The additional fourth equation describes the flow of the amplitude $\mathcal{C}$ of the correlated disorder under renormalization. The terms proportional to $A_{d}$ are the result of a firstorder expansion in $\bar{\lambda}^{2}$. In this approximation there is no influence of the correlated disorder on the renormalization of $\lambda, \nu$, and $\mathcal{D}$. Without the first-order corrections, Eqs. (6)-(9) just express the scaling relations (see also Sec. II). For the known values of the exponents $\chi(d)$ and $z(d)$, we have $(d / d l) \mathcal{C}>0$ for both $d=1$ and 2. This means that $\sigma$ will grow under renormalization, and will become of the same order of magnitude as the nonlinear $\lambda$ term at some scale $r_{c}$. For scales larger than $r_{c}$ the (first order) RG approach is no longer applicable. The above considerations show that $\sigma$ is a relevant variable. On large length scales it determines the behavior of the system in both two and one dimensions.

Scaling analysis: To obtain results outside the range of validity of the RG approach we employ the phenomenological scaling analysis (see, Refs. 23, 22, and 17). Consider a region with characteristic scale $r$. From Eq. (4) we conclude that $h$ scales with $r$ as $h \propto r^{\chi}$, and the nonlinear term in the modified KPZ equation [Eq. (1)] as $|\nabla h|^{2} \propto r^{2 \chi^{-2}}$. According to Eq. (3), the integral of the correlator $\overline{\sigma(\mathbf{x}) \sigma\left(\mathbf{x}^{\prime}\right)}$ over the interface is proportional to $\vartheta$. Hence the disorder field $\sigma(\mathbf{x})$ scales with the distance $r$ as $\sigma \propto \vartheta^{1 / 2} r^{-d / 2}$, i.e., it scales with a different exponent than the nonlinear term in Eq. (1). Without correlated disorder, $\chi$ takes the values $\chi=\frac{1}{2}$ and $\chi \simeq 0.387$ for $d=1$ and 2, respectively. Using these values in the exponent of the nonlinear term, one sees that for both dimensionalities the scaling dimension of the field $\sigma(\mathbf{x})$ is smaller than that of the nonlinear term. This indicates that for both $1 \mathrm{D}$ and 2D interfaces the correlated disorder determines the long-distance behavior of the systems.

Next we derive a useful relation between the typical fluctuation of the interface growth velocity and the concentration of correlated disorder $\vartheta$. First we find the characteristic scale $r_{c}$ at which the nonlinear term and the correlated disorder term become of the same order. This length is of the order of the size of transition region between facets. Equating the above expressions for the scale dependences of the nonlinear term and the disorder field $\sigma(\mathbf{x})$, we find

$$
r_{c} \propto \vartheta^{-1 /(4-4 \chi-d)} .
$$

The value of $r_{c}$ in Eq. (10) determines the scale of a typical fluctuation due to the $\sigma$ term in the modified KPZ equation. For a 1D interface, Eq. (10) yields $r_{c} \propto \vartheta^{-1}$. Substituting Eq. 


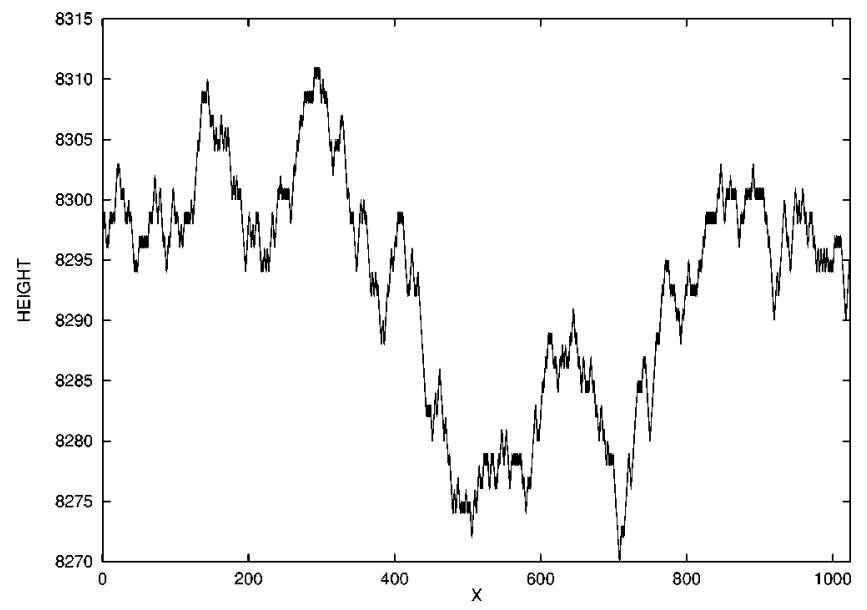

FIG. 1. Height profile $H(x)$ for periodically distributed correlated defects with concentration $\vartheta=\frac{1}{2}$, and growth probability $p=\frac{1}{2}$.

(10) into Eq. (1), we find the magnitude of the typical fluctuation of the interface growth velocity:

$$
\delta V \propto \vartheta^{1 / 2} r_{c}^{-d / 2} \propto \vartheta^{2(1-\chi) /(4-4 \chi-d)} .
$$

The fluctuation $\delta V$ is caused by the $\sigma$ field, i.e., it is due to time-independent fluctuations in the distribution of the defects. For a 1D interface, Eq. (11) predicts that $\delta V \propto \vartheta$. We use this relation to check the results of our numerical simulation.

In earlier work, ${ }^{23}$ the above phenomenological scaling approach was applied to the problem of flux line localization in 2D systems with competing thermal fluctuations and linear disorder. The results obtained ${ }^{23}$ in this manner are confirmed by exact calculations for the problem of localization of a 1D quantum particle in a random potential (see Ref. 24).

\section{NUMERICAL SIMULATION}

We have studied the inhomogeneous surface growth in $(1+1)$ dimensions numerically. Simulations were performed on the single-step model $^{3}$ using multisite coding techniques. The growth process is implemented by selecting the sites of the 1D lattice on which the particles are deposited from a sufficiently large set of random numbers. Correlated defects are introduced by taking the growth rate $p$ to be 1 on a fraction $\vartheta$ of randomly chosen sites of the 1D lattice while $p$ is varied through the values $p=\frac{1}{8}, \frac{1}{4}, \frac{1}{2}, \frac{3}{4}, \frac{7}{8}$ for the remaining lattice sites. The defect concentrations which have been considered are $\vartheta=\frac{1}{32}, \frac{1}{16}, \frac{1}{8}, \frac{1}{4}, \frac{1}{2}, \frac{3}{4}, \frac{7}{8}$, and $\frac{15}{16}$. The simulation time $t$ (number of updates per site in the growth model), is typically between $10^{4}$ and $4 \times 10^{6}$ in our simulations of the faceting and between $10^{4}$ and $10^{5}$ in our calculations of the mean facet slope. The length of the system, i.e., the number of growth points, is typically between $10^{3}$ and $3.2 \times 10^{4}$, and up to $10^{6}$ in exceptional cases.

In Fig. 1 we show the profile height $H(x)$ for a periodic array of defects with concentration $\vartheta=\frac{1}{2}$. The data have been generated with a growth rate $p=\frac{1}{2}$ on the nondefect sites. Figure 2 shows the profile height $H(x)$ obtained under the

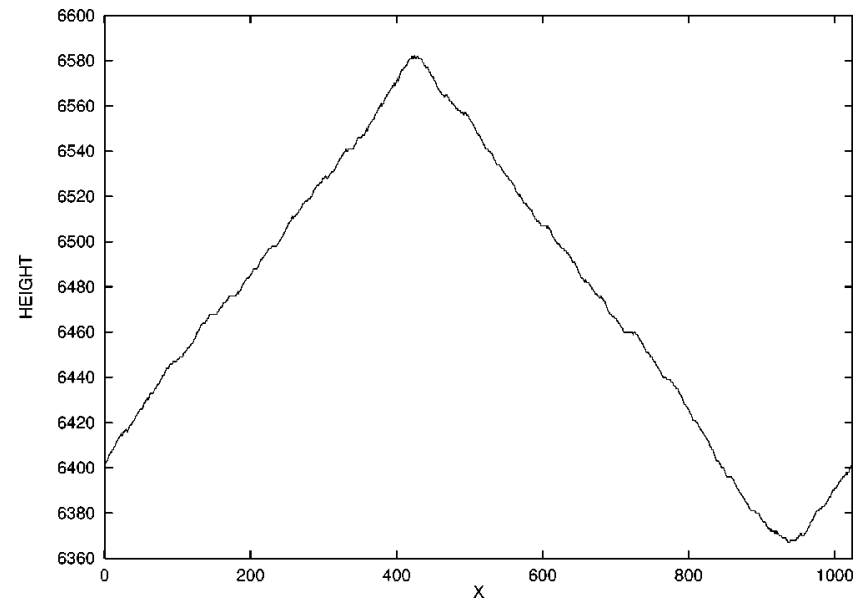

FIG. 2. Height profile $H(x)$ for randomly distributed correlated defects with the same concentration $\vartheta=\frac{1}{2}$, and the same growth probability $p=\frac{1}{2}$ as in Fig.1. The height profile is averaged over 16 realizations of stochastic growth.

same growth conditions $\left(\vartheta=\frac{1}{2}, p=\frac{1}{2}\right)$, but for a random array of correlated defects. The profile shown in Fig. 2 is an average over 16 realizations of stochastic growth. By comparing Figs. 1 and 2, the effect of randomness becomes obvious: it induces facet formation in the height profile.

Figure 3 displays the deviation $\Delta H(x)$ of the profile height $H(x)$ from its mean value $\bar{H}(x)$, for one particular realization of the correlated disorder averaged over runs with different realizations of stochastic growth (i.e., with different sets of random numbers) for three different times, namely, for $t_{1}=1.6 \times 10^{4}$ (dotted line) (averaged over 128 independent runs), $t_{2}=1.28 \times 10^{5}$ (dashed line) (averaged over 16 independent runs), and $t_{3}=10^{6}$ (continuous line) (averaged over four independent runs). As before, the growth rate on the nondefect sites is $p=\frac{1}{2}$, and the defect concentration is $\vartheta=\frac{1}{2}$. The fluctuation in $\Delta H(x)$ decreases with increasing

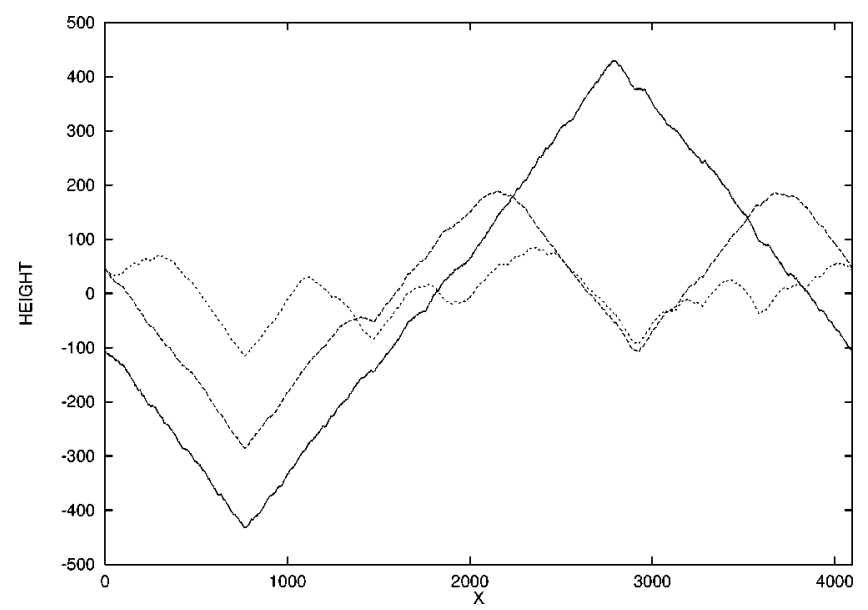

FIG. 3. Height profile $\Delta H(x)$ for one realization of linear dis$\operatorname{order}\left(\vartheta=\frac{1}{2}, p=\frac{1}{2}\right)$ and different length of the runs: $t=1.6 \times 10^{4}$ (dotted line) (averaged over 128 realizations of stochastic growth) for $t=1.28 \times 10^{5}$ (dashed line) (averaged over 16 realizations of stochastic growth) and for $t=10^{6}$ (continuous line) (averaged over four realizations of stochastic growth). 


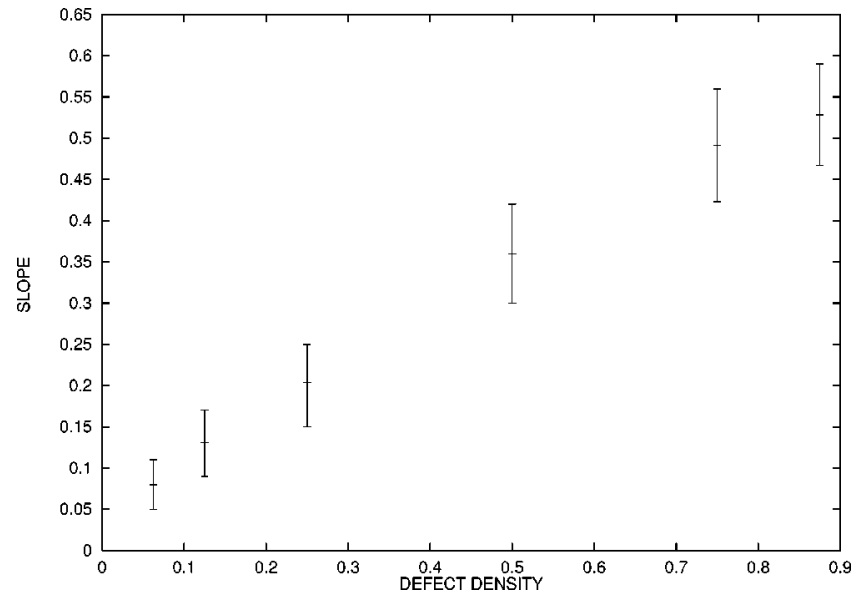

FIG. 4. Average facet slope $\varepsilon_{p}$ as a function of concentration $\vartheta$ of correlated disorder for $p=\frac{1}{2}$.

simulation times, so that only a few realizations of stochastic growth (independent runs) are necessary for $t=10^{6}$. In Fig. 3 it is seen that for sufficiently long simulation times the typical size of the transition region between the facets is much smaller than the distance between neighboring profile minima. This is true for all values of $p$ and $\vartheta$ which we have considered.

The results shown in Fig. 3 suggest that $\Delta H(x)$ increases approximately linearly with the distance from a local height minimum. Furthermore, in the vicinity of a local height minimum the slope $\varepsilon_{p}=d \Delta H\left(x_{0}\right) / d x_{0}$ of the $\Delta H(x)$ curves does not vary significantly with the simulation time $t$ or the total height $H$. To check these features, we computed the mean facet slope $\varepsilon_{p}$ different growth rates $p$ and for different concentrations $\vartheta$. For given values of $p$ and $\vartheta$ the simulation has been repeated for up to 512 realizations of the correlated disorder. For each realization of the disorder an average over up to 64 independent growth runs (i.e., runs with independent sets of random numbers) has been taken. For fixed values of the growth rate $p$ the mean facet slope $\varepsilon_{p}$ varies approximately linearly with the concentration $\vartheta$ in the limit of small $\vartheta$. This is seen in Fig. 4, where we show the average facet slope $\varepsilon_{p}$ as a function of the defect concentration $\vartheta$ from simulations with the values of growth probability $p=\frac{1}{2}$. Figure 5 displays a further check on the validity of the linear dependence of the average facet slope on the defect concentration, $\varepsilon_{p}(\vartheta) \propto \vartheta$. Values for $\varepsilon_{p}(2 \vartheta)$ and $\varepsilon_{p}(\vartheta)$ obtained from simulations with growth rates $p=\frac{1}{2}, \frac{1}{4}, \frac{1}{8}$, and $\frac{3}{4}$ for the nondefect sites are plotted against each other. The data collapse on the theoretically predicted line $\varepsilon_{p}(2 \vartheta)=2 \varepsilon_{p}(\vartheta)$ is satisfactory.

The simulations reveal a qualitative picture of the faceting of the 1D interface. A typical fluctuation in the distribution of the correlated defects creates a transition region between facets, i.e., a local minimum in the height profile, with a typical width $r_{c}$. Suppose that at a time $t_{0}$ there are only two height minima with distance $2 X_{0}$ between them. The lower one has the lower growth rate. Thus, the height difference between two minima will grow with time $t$ until the height difference reaches the maximum value compatible with $\varepsilon_{p}(\vartheta) X_{0}$. At this moment of time $\left(t_{1}\right)$ the higher minimum will simply disappear. One can repeat this Gedanken experi-

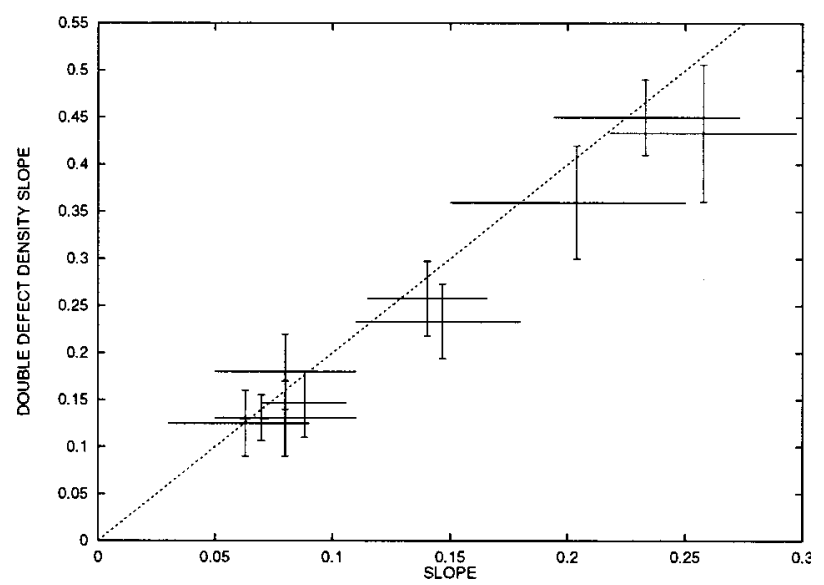

FIG. 5. Average facet slope $\varepsilon_{p}(2 \vartheta)$ at double concentration $2 \vartheta$ of correlated disorder vs $\varepsilon_{p}(\vartheta)$ for different values of the parameter $p$ (growth rate): $p=\frac{1}{2}, \frac{1}{4}, \frac{1}{8}, \frac{3}{4}, \frac{7}{8}$.

ment for any number of minima, with the result that with increasing time the number of minima decreases. Figure 3 nicely illustrates this faceting process. We assume that the velocity difference $\Delta V$ between two points is almost constant, and is of the order of the typical velocity fluctuation: $\Delta V \approx \delta V$. Then $\varepsilon_{p} X_{0} \approx \delta V\left(t_{1}-t_{0}\right)$. In our numerical model the units of time and distance are the same, i.e., $X_{0}=t_{1}-t_{0}$ and $\delta V \approx \varepsilon_{p} \propto \vartheta$. This supports the results of our renormalization group and scaling analysis. Indeed, according to Eq. (11) the typical variation of the growth velocity $\delta V$ is proportional to $\vartheta$.

\section{CONNECTION TO FLUX LINE LOCALIZATION}

The problem of a growing interface profile can be mapped onto the problem of the ground state of a directed polymer or of a flux line (FL) in a disordered medium. The modified Kardar-Parizi-Zhang equation, Eq. (1), can be transformed into the imaginary-time Schrödinger equation

$$
\frac{\partial W(\mathbf{x}, t)}{\partial t}=\nu \Delta W(\mathbf{x}, t)+\frac{[\eta(\mathbf{x}, t)+\sigma(\mathbf{x})]}{\lambda} W(\mathbf{x}, t),
$$

where the "wave function" has the form

$$
W(\mathbf{x}, t)=\exp \left(-\frac{\lambda}{\nu} h(\mathbf{x}, t)\right) .
$$

The solution of Eq. (12) can be written as

$$
\begin{aligned}
W(\mathbf{x}, t)= & \int_{0,0}^{\mathbf{x}, t} \mathcal{D} \mathbf{y} \\
& \times \exp \left\{-\frac{1}{2 \nu} \int_{0}^{t} d z\left[\frac{1}{2}\left(\frac{d \mathbf{y}}{d z}\right)^{2}-\lambda[\eta(\mathbf{y}, z)+\sigma(\mathbf{y})]\right]\right\}
\end{aligned}
$$

After a suitable change of the independent variables Eq. (14) can be considered as the partition function of a system described by the Hamiltonian 


$$
\mathcal{H}=\int d z\left[\frac{\boldsymbol{\epsilon}}{2}\left(\frac{d \mathbf{x}}{d z}\right)^{2}+\eta(\mathbf{x}, z)+\sigma(\mathbf{x})\right] .
$$

Here $\mathbf{x}(z)$ can be alternatively interpreted as the position vector of a directed polymer or of a flux line as a function of $z$. $\epsilon$ is the line tension, $\eta(\mathbf{x}, z)$ is a quenched random point potential, and $\sigma(\mathbf{x})$ is a correlated (independent on $z$ ) random potential. The point and correlated (linear) random potentials are assumed to be Gaussian distributed, with mean zero and with correlators as defined in Eq. (3). In this mapping the mean value of the height $\langle h(\mathbf{x}, t)\rangle$ corresponds to the free energy of the polymer or of the flux line:

$$
\langle h(\mathbf{x}, t)\rangle=-\left\langle\frac{\nu}{\lambda} \ln W(\mathbf{x}, t)\right\rangle .
$$

We briefly discuss the mapping of the results obtained in Sec. IV in terms of flux line localization. First, we consider the mapping rules for the case of numerical simulations. In this mapping the growth rate, $p$ corresponds to the relative strength of the correlated disorder and the point disorder. Small values of $p$ correspond to the relatively strong correlated disorder, and small values of $(1-p)$ correspond to weak correlated disorder. ${ }^{5,17}$ The length $L$ of the FL, measured in units of updates per site, corresponds to the time in the growth model.

Without the last term the Hamiltonian, Eq. (15), describes the fluctuations of a single FL subject only to point disorder. This problem has been studied in detail (see e.g., Ref. 20). The energy per unit length $\varepsilon_{p d}$ of a FL scales with its transverse extension $r$ as $\varepsilon_{p d} \propto r^{2-2 / \zeta}$, where the roughening exponent $\zeta(\zeta=1 / z)$ is known exactly in $d=1[\zeta=2 / 3$ (Ref. 20)] and numerically in $d=2[\zeta=0.620$ (Ref. 21)]. To describe FL localization phenomena we repeat the phenomenological scaling analysis used above (see Refs. 23, 22, and 17). For the case of correlated disorder the interaction energy per unit length of the FL $\varepsilon_{l d}$ scales with the transverse extension $r$ as $\varepsilon_{l d} \propto \sqrt{\mathcal{C}} r^{-d / 2}$. Both $\varepsilon_{p d}$ and $\varepsilon_{l d}$ scale with $r$ with the same exponent at some dimensionality $d_{c}$. Comparing $\varepsilon_{p d}$ and $\varepsilon_{l d}$ one finds for the dimensionality $d_{c}$ $=4\left[1-\zeta\left(d_{c}\right)\right] / \zeta\left(d_{c}\right)$. For dimensionalities $d<d_{c}$ a single FL is localized by typical fluctuations of the correlated disorder. From the same scaling relations, for the localization length one obtains $r_{c} \propto \vartheta \tilde{\gamma}$, where $\tilde{\gamma}=2 /(4+d-4 / \zeta)$. The localization length corresponds to the width of the transition region between facets in the growth problem. For the pinning energy one has $\varepsilon_{p} \propto \vartheta^{\beta}$ where $\beta=d \gamma / 2=2 d(1$ $-1 / \zeta) /(4+d-4 / \zeta)$, and $\beta=1$ for $d=1$, and $\beta=5.42$ for $d=2$. The pinning energy corresponds to the slope of the height profile in the growth problem. For pure thermal fluctuation with $\zeta=\frac{1}{2}$, one has $\beta=2 d /(4-d)$, and $d_{c}=4 .^{22}$ In the limit of vanishing strength of the correlated disorder, on a large scale the above result does not depend on the details of the disorder potential. ${ }^{25}$

The simulation yields a qualitative picture of FL localization. A typical fluctuation in the distribution of the linear defects localizes the FL in a strip with width $r_{c}$. The energies of such typical energy minima are distributed with some density $\rho(E)$, and among them there is a lowest one, the absolute minimum (the energy spectrum is bounded from below). The variation of the FL energy $V(x)$ of a short FL reflects this energy spectrum. Indeed, the energy difference between two localized states grows linearly with the FL length, and at some critical length it will be larger than the additional energy need to overcome the barrier between these localized states. This process manifests itself in Fig. 3: the number of pronounced minima of $V(x)$ decreases as the length of the FL increases, until only the state with lowest energy survives (see Fig. 3).

\section{DISCUSSION}

The following comments concern experimental realizations of the model systems discussed in the previous sections.

Magnetization reversal in ultrathin magnetic films: The magnetic degrees of freedom of ultrathin magnetic films can be modelled by the Ising model. Domain growth in the Ising model has been discussed by many authors, ${ }^{12-14}$ and it has been shown that the single-step model applies to this problem for low temperatures. In this case steps with fluctuating distance between them can play the role of the correlated disorder. The magnetic domain imaging technique (see, e.g., Refs. 26 and 27) has a resolution of up to $20 \mathrm{~nm}$ which is comparable with domain-wall width. Therefore, with this technique it should be possible study the influence of correlated disorder (steps) on the domain boundary profile, and to check our prediction of domain boundary faceting.

Faceting of a two-dimensional interface: Numerical simulations have been performed for the case of a onedimensional interface only. However, the qualitative picture of faceting phenomena developed in Sec. IV applies to both one- and two-dimensional interfaces. Therefore we expect the faceting phenomenon also to occur in the growth of a two-dimensional surface with correlated defects. One of the possible experimental realization of correlated disorder is a nanoparticle on the growing surface. In fact, in their study of the growth of a $\mathrm{Si}(001)$ surface, the authors of Refs. 18 and 19 observed the formation of pyramids consisting of four facets on the background of a roughly growing surface. The formation of pyramids has been attributed to nanosize defects created during the growth procedure.

Diffusion and faceting: Stochastic growth models neglect the lateral diffusion. We outline briefly the conditions under which the faceting phenomenon remains in the presence of lateral diffusion. One can neglect lateral diffusion if the flux of particles due to lateral diffusion is much smaller than flux due to the growth process. Suppose that the driving force in the lateral diffusion is proportional to the surface slope $\varepsilon$. Then from the Einstein relation the corresponding current $J_{l} \propto\left(D_{l} / T\right) \varepsilon$. The incoming flux $J_{G} \approx p+(1-p) \vartheta$. The condition of the validity of the stochastic growth assumption is $J_{G} \gg J_{l}$. The diffusion constant $D=D_{0} \exp \left(-E_{a} / T\right)$, where $E_{a}$ is the diffusion activation energy. By changing the temperature one can always tune to the stochastic growth regime.

\section{CONCLUSION}

To conclude, we have shown that, during the growth or evaporation of an initially flat surface, large-scale faceting develops under the influence of disorder which is correlated 
in the direction normal to the surface. For a sample of arbitrary size only two (for a 1D interface) or four (for a 2D interface with cubic symmetry) facets survive after a sufficiently long time. In the latter case the interface has a pyramid-shaped profile. The phenomenological scaling analysis in combination with numerical simulations provides a simple description of the faceting phenomenon, and it is predicted to occur for dimensionalities $d=1$ and 2. Owing to the connection between the models describing interface growth in $d$ dimensions on the one hand, and the groundstate conformation of a directed polymer or of a flux line in a superconductor in $d+1$ dimensions on the other, we can conclude that these last systems are localized in the presence of both point disorder and correlated disorder.

\section{ACKNOWLEDGMENTS}

We benefited from discussions with L.-H. Tang, G.V. Uimin, and T. Nattermann. We wish especially to thank L.-H. Tang for help with simulation. The work was supported by the Niedersächsische Ministerium für Wissenschaft and Kultur. One of us (I.F.L.) was partly supported by SFB 341, by U.S. DOE Grant No. DE-FG03-96ER 45598 and by NSF Grant No. DMR 9705182.
*Also at Institute of Physics, $252028 \mathrm{Kiev}$, Ukraine.

${ }^{1}$ E. Bauer, Z. Kristallogr. 110, 372 (1958).

${ }^{2}$ M. Kardar, G. Parisi, and Y. C. Zhang, Phys. Rev. Lett. 56, 889 (1986).

${ }^{3}$ P. Meakin, P. Ramanlal, L. M. Sander, and R. C. Ball, Phys. Rev. A 34, 5091 (1986).

${ }^{4}$ M. Plischke, Z. Racz, and D. Liu, Phys. Rev. B 35, 3485 (1987).

${ }^{5}$ L.-H. Tang, in Growth Patterns in Physical Sciences and Biology, edited by J. M. Garcia-Ruiz, E. Louis, P. Meakin, and L. M. Sander (Plenum, New York, 1991), p. 99.

${ }^{6} \mathrm{D}$. Wolf, in Scale Invariance, Interfaces, and Non-Equilibrium Dynamics, edited by A. McKane, M. Droz, J. Vannimenus, and D. Wolf (Plenum, New York, 1995), p. 215.

${ }^{7}$ J. Krug, in Scale Invariance, Interfaces, and Non-Equilibrium Dynamics (Ref. 6), p. 1.

${ }^{8}$ M. Henzler, M. Horn-von Hoegen, and U. Köhler, Nova Acta Leopoldina NF 73, 31 (1995).

${ }^{9}$ M. Henzler, T. Schmidt, and E. Z. Luo, in Proceedings of the IV International Conference on the Structure of Surfaces, edited by H. Schmidt (World Scientific, Singapore, 1994), p. 619.

${ }^{10}$ E. Z. Luo, J. Wollschläger, F. Wegner, and M. Henzler, Appl. Phys. A: Solids Surf. 60, 19 (1995).

${ }^{11}$ U. Kdohler, L. Andersohn, and B. Dahlheimer, Appl. Phys. A: Solids Surf. 57, 491 (1993).

${ }^{12}$ J.-P. Marchand and Ph. A. Martin, J. Stat. Phys. 44, 491 (1986).

${ }^{13}$ D. Kandel and E. Domany, J. Stat. Phys. 58, 685 (1990).

${ }^{14}$ J. Krug and H. Spohn, in Solids Far From Equilibrium, edited by
C. Godreche (Cambridge University Press, Cambridge 1991).

${ }^{15}$ I. Lyuksyutov, A. G. Naumovets, and V. L. Pokrovsky, TwoDimensional Crystals (Academic, Boston, 1992).

${ }^{16}$ J.-S. Suen and J. L. Erskine, Phys. Rev. Lett. 78, 3567 (1997).

${ }^{17}$ L.-H. Tang and I. F. Lyuksyutov, Phys. Rev. Lett. 71, 2745 (1993).

${ }^{18}$ F. Meyer zu Heringdorf, H. Goldbach, and H.-L. Günter, Verhandlungen der DPG, Früjahrestagung 1996 (Physik-Verlag, Regensburg, 1996).

${ }^{19}$ F. Meyer zu Heringdorf, Diplomarbeit, Institut für Festkörperphysik, Universität Hannover, 1995.

${ }^{20}$ D. A. Huse, C. L. Henley, and D. S. Fisher, Phys. Rev. Lett. 55, 2924 (1985); M. Kardar, Nucl. Phys. B 290, 582 (1987); E. Medina, T. Hwa, M. Kardar, and Y. C. Zhang, Phys. Rev. A 39, 3053 (1989).

${ }^{21}$ B. M. Forrest and L.-H. Tang, Phys. Rev. Lett. 64, 1405 (1990).

${ }^{22}$ I. F. Lyuksyutov, Europhys. Lett. 20, 273 (1992).

${ }^{23}$ I. F. Lyuksyutov, Zh. Eksp. Teor. Fiz. 88, 871 (1985) [Sov. Phys. JETP 61, 512 (1984)].

${ }^{24}$ I. M. Lifshits, S. A. Gredeskul, and L. A. Pastur, Introduction to the Theory of Disordered Systems (Wiley, New York, 1988).

${ }^{25}$ D. E. Wolf and L.-H. Tang, Phys. Rev. Lett. 65, 1591 (1990); and in Growth and Form: Nonlinear Aspects, edited by P. Pelce (Plenum, New York, 1991).

${ }^{26}$ R. Allenspach, J. Magn. Magn. Mater. 129, 160 (1994).

${ }^{27}$ H. Pinkvos, H. Poppa, E. Bauer, and J. Hurst, Ultramicroscopy 47, 339 (1992). 